\title{
Per-Node Based Optimal Power Control for Multi-Hop Cognitive Radio Networks
}

\author{
Yi Shi, Member, IEEE, Y. Thomas Hou, Senior Member, IEEE, and Huaibei Zhou
}

\begin{abstract}
Cognitive radio network (CRN) is a promising approach to improve spectrum efficiency for wireless networking. This paper investigates how to perform optimal power control on each node (or per-node based power control) in the network so as to optimize network performance. Per-node based power control is a difficult problem due to its large design space (i.e., interaction among the powers on different nodes in the network) and the coupling relationship between power control and upper layers (scheduling and routing). In this paper, we develop a formal mathematical model for joint power control, scheduling, and routing. We formulate a cross-layer optimization problem encompassing these three layers and develop a unified solution procedure based on branch-and-bound framework and convex hull relaxation. Using numerical results, we demonstrate the efficacy of the solution procedure and offer insights on the behavior of per-node based power control.
\end{abstract}

Index Terms-Cognitive radio network (CRN), multi-hop networking, per-node based power control, interference modeling, cross-layer optimization.

\section{INTRODUCTION}

C OGNITIVE radio $(\mathrm{CR})$ is a revolution in radio technology that is enabled by recent advances in RF design, signal processing, and communications software [18]. The capability of $\mathrm{CR}$ has been recognized by the military and commercial sector and is now under intensive research and development by the DoD's Joint Tactical Radio System (JTRS) program [10] and wireless industry [19]. Since transmitted waveform is defined by software, a CR is capable of reconfiguring RF (on the fly) and switching to newly-selected frequency bands. From networking perspective, the emergence of CR offers new challenges in algorithm and protocol design.

It is important to realize that a CR is vastly more powerful and flexible than existing multi-channel multi-radio (MC-MR) technology (see e.g., [1], [6], [11], [12], [17] and reference therein). Note that MC-MR remains hardware-based radio technology: each radio can only operate on a single channel at a time and the number of concurrent channels that can be used at a wireless node is limited by the number of radio interfaces. In addition, an MC-MR wireless network typically

Manuscript received December 26, 2008; revised May 13, 2009; accepted July 6, 2009. The associate editor coordinating the review of this paper and approving it for publication was J. Zhang.

Y. Shi and Y. T. Hou are with the Bradley Department of Electrical and Computer Engineering, Virginia Tech, Blacksburg, VA 24061, USA (e-mail: \{thou,yshi\}@vt.edu).

H. Zhou is with the International School of Software Engineering, Wuhan University, China (e-mail: bzhou@whu.edu.cn).

The work by Y. T. Hou and Y. Shi was supported in part by the U.S. National Science Foundation (NSF) under grants CNS-0831865 and CNS0721421. The work by H. Zhou was supported by the National Basic Research Program of China (973 Program) under grant 2009CB3204001.

Digital Object Identifier 10.1109/TWC.2009.081702 works on a set of pre-assigned channels and is not able to sense available spectrum. In contract, each node in a CRN is able to perform spectrum sensing and work on a different set of available frequency bands. These important differences warrant that algorithm design for a CRN is more complex and interesting.

A fundamental problem for a wireless network is power control. In a multi-hop wireless network, power control is challenging since it directly affects upper layers scheduling and routing. When each node is allowed to perform power control (which we call per-node based power control), the problem becomes even more difficult due to its large optimization space. Due to such difficulty, some previous work, e.g., [2], [12], considered synchronized power control, where transmission power at each node in the network is adjustable but is synchronized to be identical. Needless to say, such synchronization in power control cannot offer optimal network performance.

Recognizing the benefits of per-node based power control, there have been active research efforts in recent years. But pernode based power control is an extremely difficult problem as it is tightly coupled with scheduling and routing. Due to such coupling, a joint formulation of multiple layers is necessary, which inevitably lead to a very complex problem. Although there has been some success in the context of asymptotic scaling laws (e.g., [9], [12]), theoretically optimal results for a given finite-sized network remain unsatisfactory and many issues are still open. For example, in [3], Bhatia and Kodialam optimized power control and routing, but assumed some frequency hopping mechanism is in place for scheduling, which helps simplify joint consideration of scheduling. In [7], Elbatt and Ephremides optimized power control and scheduling, but assumed routing was given a priori. Although [4], [5] aimed to investigate joint power control, scheduling, and routing problem, both solutions followed a "de-coupled" approach, where the final solution was obtained by determining algorithm/mechanism for one layer at a time (instead of solving a unified problem, as we shall do in this paper). Due to such de-coupling approach in solution procedure, the final solution is unlikely to be cross-layer globally optimal.

In this paper, we study the per-node based power control problem for a multi-hop CRN. The benefits of per-node based power control for CRNs were discussed in [22]. This problem is both challenging and interesting as it inherits not only all the difficulties associates with per-node based power control, but also all the unique characteristics associated with a CRN. We develop a formal mathematical model for a joint per-node based power control, scheduling, and flow routing problem. 
TABLE I

NOTATION.

\begin{tabular}{|c|c|}
\hline Symbol & Definition \\
\hline $\mathcal{N}$ & The set of nodes in the network \\
\hline $\mathcal{M}_{i}$ & The set of available bands at node $i \in \mathcal{N}$ \\
\hline $\mathcal{M}$ & $=\sum_{i \in \mathcal{N}} \mathcal{M}_{i}$, the set of bands in the network \\
\hline $\mathcal{M}_{i j}$ & $=\mathcal{M}_{i} \cap \mathcal{M}_{j}$, the set of available bands at link $i \rightarrow j$ \\
\hline$W$ & Bandwidth of each frequency band \\
\hline $\mathcal{L}$ & The set of user communication sessions \\
\hline$s(l), d(l)$ & Source and destination nodes of session $l \in \mathcal{L}$ \\
\hline$r(l)$ & Rate requirement of session $l$ \\
\hline$P$ & The maximum transmission power \\
\hline$n$ & Path loss index \\
\hline$\eta$ & Ambient Gaussian noise density \\
\hline$d_{i j}$ & Distance between nodes $i$ and $j$ \\
\hline$g_{i j}$ & Path attenuation loss from node $i$ to node $j$ \\
\hline$\alpha$ & The threshold of minimum receiving power \\
\hline$\beta$ & The threshold of maximum interference power \\
\hline$R_{T}(p), R_{I}(p)$ & $\begin{array}{l}\text { The transmission and interference ranges under } \\
\text { transmission power } p\end{array}$ \\
\hline$R_{T}^{\max }, R_{I}^{\max }$ & $\begin{array}{l}\text { The maximum transmission and interference ranges } \\
\text { under full transmission power } P\end{array}$ \\
\hline $\mathcal{T}_{i}^{m}$ & $\begin{array}{l}\text { The set of nodes to which node } i \text { can transmit on } \\
\text { band } m \text { (under full transmission power } P \text { ) }\end{array}$ \\
\hline $\mathcal{T}_{i}$ & $\begin{array}{l}=\bigcup_{m \in \mathcal{M}_{i}} \mathcal{T}_{i}^{m} \text {, the set of nodes to which node } i \\
\text { can transmit (under full transmission power } P \text { ) }\end{array}$ \\
\hline $\mathcal{I}_{j}^{m}$ & $\begin{array}{l}\text { The set of nodes that can interfere node } j \text { on band } \\
m \text { (under full transmission power } P \text { ) }\end{array}$ \\
\hline$x_{i j}^{m}$ & $\begin{array}{l}\text { Binary indicator to mark whether or not band } m \text { is } \\
\text { used by link } i \rightarrow j\end{array}$ \\
\hline$p_{i j}^{m}$ & Transmission power at node $i$ to node $j$ on band $m$ \\
\hline$c_{i j}^{m}$ & Link capacity of link $i \rightarrow j$ under $p_{i j}^{m}$ \\
\hline$f_{i j}(l)$ & Data rate that is attributed to session $l$ on link $i \rightarrow j$ \\
\hline$Q$ & Number of transmission power levels \\
\hline$q_{i j}^{m}$ & $\begin{array}{l}\text { Transmission power level from node } i \text { to node } j \text { on } \\
\text { band } m\end{array}$ \\
\hline $\mathbf{x}$ & The vector of variables $x_{i j}^{m}, i \in \mathcal{N}, m \in \mathcal{M}_{i}, j \in \mathcal{T}_{i}^{m}$ \\
\hline $\mathbf{q}$ & The vector of variables $q_{i j}^{m}, i \in \mathcal{N}, m \in \mathcal{M}_{i}, j \in \mathcal{T}_{i}^{m}$ \\
\hline f & $\begin{array}{l}\text { The vector of variables } f_{i j}(l), l \in \mathcal{L}, i \in \mathcal{N}, i \neq d(l) \text {, } \\
j \in \mathcal{T}_{i}, j \neq s(l)\end{array}$ \\
\hline \multicolumn{2}{|r|}{ Notation for branch-and-bound procedure } \\
\hline$\varepsilon$ & The desired accuracy in the final solution \\
\hline$\Omega_{z}$ & The set of all possible values of $(\mathbf{x}, \mathbf{q})$ in problem $z$ \\
\hline$L B_{z}, U B_{z}$ & The lower and upper bounds of problem $z$ \\
\hline$\psi_{z}$ & The solution obtained by local search for problem $z$ \\
\hline $\begin{array}{l}L B, U B \\
\psi_{\varepsilon}\end{array}$ & $\begin{array}{l}\text { The lower and upper bounds for the original problem } \\
\text { The }(1-\varepsilon) \text { optimal solution }\end{array}$ \\
\hline
\end{tabular}

This joint formulation leads to a mixed integer nonlinear programming (MINLP) problem. Subsequently, we develop a unified (instead of de-coupled) solution procedure based on branch-and-bound framework and convex hull relaxation. This solution procedure guarantees a $(1-\varepsilon)$ optimal solution, where $\varepsilon$ is a small pre-specified error tolerance parameter. By applying the solution procedure on a random network, we validate this solution procedure and offer additional insights on the behavior of per-node based power control. The results in this paper close some long stand open issues associated with network optimization with per-node based power control.

The remainder of this paper is organized as follows. In Section II, we develop a unified mathematical model for pernode based power control, scheduling, and flow routing. In Section III, we formulate the cross-layer optimization problem. Section IV describes a solution procedure to this crosslayer optimization problem. In Section V, we use numerical results to validate the efficacy of the solution procedure. Section VI concludes this paper.

\section{Mathematical Modeling}

We consider a multi-hop CRN consisting of a set of $\mathcal{N}$ nodes. The set of available frequency bands at each node depends on its location and may not be the same. For example, at node $i$, its available frequency bands may consist of bands I, III, and V while at a different node $j$, its available frequency bands may consist of bands I, IV, and VI, and so forth. More formally, denote $\mathcal{M}_{i}$ the set of available frequency bands at node $i$. For simplicity, we assume the bandwidth of each frequency band is $W$ (the case of heterogeneous bandwidth for each frequency band can be easily extended). Denote $\mathcal{M}$ the set of all frequency bands present in the network, i.e., $\mathcal{M}=\bigcup_{i \in \mathcal{N}} \mathcal{M}_{i}$. Table I lists all notation in this paper.

\section{A. Necessary and Sufficient Condition for Successful Trans- mission}

Scheduling for transmission at each node in the network can be done either in time domain or frequency domain and these two scheduling schemes are equivalent in terms of achievable rate region. In this paper, we consider scheduling in the frequency domain in the form of frequency bands. Note that in this context, we can always divide bands into smaller sub-bands to ensure there are enough bands/sub-bands for scheduling.

Suppose that band $m$ is available at both node $i$ and node j, i.e., $m \in \mathcal{M}_{i j}$, where $\mathcal{M}_{i j}=\mathcal{M}_{i} \cap \mathcal{M}_{j}$. Denote $p_{i j}^{m}$ the transmission power from node $i$ to node $j$ in frequency band $m$. For transmission from node $i$ to node $j$, a simple model for path attenuation loss $g_{i j}$ is

$$
g_{i j}=d_{i j}^{-n},
$$

where $d_{i j}$ is the physical distance between nodes $i$ and $j$ and $n$ is the path loss index. In this context, we assume a data transmission from node $i$ to node $j$ is successful only if the received power at node $j$ exceeds a threshold $\alpha$. Denote the transmission range of at node $i$ under $p_{i j}^{m}$ as $R_{T}\left(p_{i j}^{m}\right)$. Then based on $g_{i j} \cdot p_{i j}^{m} \geq \alpha$ and (1), we can calculate the transmission range of this node as follows:

$$
R_{T}\left(p_{i j}^{m}\right)=\left(\frac{p_{i j}^{m}}{\alpha}\right)^{1 / n} .
$$

Since the receiving node $j$ must be physically within the transmission range of node $i$, we have

$$
d_{i j} \leq\left(\frac{p_{i j}^{m}}{\alpha}\right)^{1 / n} .
$$

Similarly, we assume that an interference is non-negligible only if it exceeds a threshold, say $\beta$ at a receiver. Denote the interference range of node $k(k \in \mathcal{N}, k \neq i)$ under $p_{k h}^{m}$ as $R_{I}\left(p_{k h}^{m}\right)$, where $h$ is the intended receiving node of transmitting node $k$. Then following the same taken as the derivation for the transmission range, we can obtain the interference range of node $k$ as $R_{I}\left(p_{k h}^{m}\right)=\left(\frac{p_{k h}^{m}}{\beta}\right)^{1 / n}$. Since the receiving node $j$ must not fall in the interference range of any other node $k$ that is transmitting in the same band, we have

$$
d_{j k} \geq\left(\frac{p_{k h}^{m}}{\beta}\right)^{1 / n}
$$




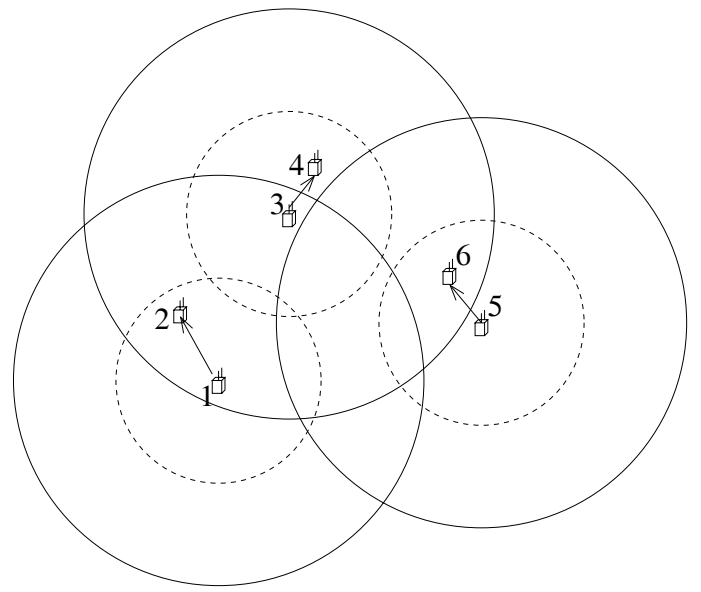

(a) No power control case.

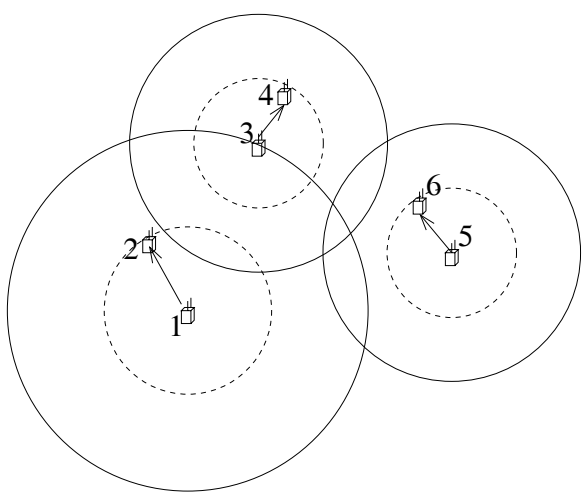

(b) Per-node based power control case.

Fig. 1. A 3-link network.

As an example, Fig. 1(a) shows a network with three links $(1 \rightarrow 2,3 \rightarrow 4$, and $5 \rightarrow 6$ ). For each transmitting node $(1,3$, and 5$)$, the inner circle (dashed) represents transmission range and the outer circle (solid) represents interference range. Clearly, each receiving node falls in the transmission range of its respective transmitting node. Further, we can see that both receiving nodes 2 and 6 fall in the interference range of node 3 . Thus, when link $3 \rightarrow 4$ is using a frequency band $m$ for transmission, link $1 \rightarrow 2$ and link $5 \rightarrow 6$ should not use the same band. It should also be noted that when link $3 \rightarrow 4$ is not using a frequency band $m$, both link $1 \rightarrow 2$ and link $5 \rightarrow 6$ may use band $m$. This is because that receiving node 2 does not fall in node 5 's interference range and receiving node 6 does not fall in node 1's interference range. Now consider that each node can adjust its transmission power. In this setting, nodes 1,3 , and 5 can reduce their transmission powers while still maintaining data transmission to the corresponding receiving nodes (see Fig. 1(b)). Then receiving nodes 2 and 6 are no longer in node 3's interference range. As a result of this, both transmitting nodes 1 and 5 can transmit in the same frequency band $m$ simultaneously.

\section{B. Per-Node Based Power Control and Scheduling}

In this section, we formalize a mathematical model for the joint relationship between per-node based power control and scheduling. Suppose that band $m$ is available at both node $i$ and node $j$. Denote

$x_{i j}^{m}= \begin{cases}1 & \text { If node } i \text { transmits data to node } j \text { on band } m, \\ 0 & \text { otherwise. }\end{cases}$

As mentioned earlier, we consider scheduling in the frequency domain and thus once a band $m \in \mathcal{M}_{i}$ is used by node $i$ for transmission to node $j$, this band cannot be used again by node $i$ to transmit to a different node. That is,

$$
\sum_{j \in \mathcal{T}_{i}^{m}} x_{i j}^{m} \leq 1
$$

where $\mathcal{T}_{i}^{m}$ is the set of nodes to which node $i$ can transmit on band $m$ under full power $P$.

Denote $R_{T}^{\max }$ the maximum transmission range of a node when it transmits at full power $P$. Then based on (2), we have $R_{T}^{\max }=R_{T}(P)=\left(\frac{P}{\alpha}\right)^{1 / n}$. Thus, we have $\alpha=\frac{P}{\left(R_{T}^{\max }\right)^{n}}$. Then for a node transmitting at a power $p \in[0, P]$, its transmission range is

$$
R_{T}(p)=\left(\frac{p}{\alpha}\right)^{1 / n}=\left[\frac{p\left(R_{T}^{\max }\right)^{n}}{P}\right]^{1 / n}=\left(\frac{p}{P}\right)^{1 / n} R_{T}^{\max } .
$$

Similarly, denote $R_{I}^{\max }$ the maximum interference range of a node when it transmits at full power $P$. Then following the same token, we have $R_{I}^{\max }=R_{I}(P)=\left(\frac{P}{\beta}\right)^{1 / n}$ and $\beta=\frac{P}{\left(R_{I}^{\text {max }}\right)^{n}}$. For a node transmitting at a power $p \in[0, P]$, its interference range is

$$
R_{I}(p)=\left(\frac{p}{P}\right)^{1 / n} R_{I}^{\max } .
$$

Recall that $\mathcal{T}_{i}{ }^{m}$ denotes the set of nodes to which node $i$ can transmit on band $m$ under full power $P$. More formally, we have $\mathcal{T}_{i}^{m}=\left\{j: d_{i j} \leq R_{T}^{\max }, j \neq i, m \in \mathcal{M}_{j}\right\}$. Similarly, denote $\mathcal{I}_{j}^{m}$ the set of nodes that can make interference on node $j$ on band $m$ under full power $P$, i.e., $\mathcal{I}_{j}^{m}=\left\{k: d_{j k} \leq\right.$ $\left.R_{I}^{\max }, m \in \mathcal{M}_{k}\right\}$. Note that the definitions of $\mathcal{T}_{i}^{m}$ and $\mathcal{I}_{j}^{m}$ are both based on full transmission power $P$. When power level $p$ is below $P$, the corresponding transmission and interference ranges will be smaller. As a result, it is necessary to keep track of the set of nodes fall in the transmission range and the set of nodes that can produce interference whenever transmission power changes at a node.

Recall the two constraints (C-1) and (C-2) for successful transmission from node $i$ to node $j$ and (3) and (4), respectively, we have

$$
\begin{aligned}
& d_{i j} \leq R_{T}\left(p_{i j}^{m}\right)=\left(\frac{p_{i j}^{m}}{P}\right)^{1 / n} R_{T}^{\max }, \\
& d_{j k} \geq R_{I}\left(p_{k h}^{m}\right)=\left(\frac{p_{k h}^{m}}{P}\right)^{1 / n} R_{I}^{\max } \quad\left(k \in \mathcal{I}_{j}^{m}, k \neq i, h \in \mathcal{T}_{k}^{m}\right) .
\end{aligned}
$$

Based the above two constraints, we have the following requirements for the transmission link $i \rightarrow j$ and interfering link $k \rightarrow h$ :

$$
p_{i j}^{m} \begin{cases}\in\left[\left(\frac{d_{i j}}{R_{T}^{m a x}}\right)^{n} P, P\right] & \text { If } x_{i j}^{m}=1, \\ =0 & \text { If } x_{i j}^{m}=0 .\end{cases}
$$

$$
p_{k h}^{m} \leq\left\{\begin{array}{ll}
\left(\frac{d_{k j}}{R_{I}^{m a x}}\right)^{n} P & \text { If } x_{i j}^{m}=1, \\
P & \text { If } x_{i j}^{m}=0 .
\end{array} \quad\left(k \in \mathcal{I}_{j}^{m}, k \neq i, h \in \mathcal{T}_{k}^{m}\right) .\right.
$$


Mathematically, these requirements can be re-written as

$$
\begin{aligned}
& \left(\mathrm{C}-1^{\prime}\right) \quad p_{i j}^{m} \in\left[\left(\frac{d_{i j}}{R_{T}^{\text {max }}}\right)^{n} P x_{i j}^{m}, P x_{i j}^{m}\right], \\
& \left(\mathrm{C}-2^{\prime}\right) \quad p_{k h}^{m} \leq P-\left[1-\left(\frac{d_{k j}}{R_{I}^{\text {max }}}\right)^{n}\right] P x_{i j}^{m} \\
& \left(k \in \mathcal{I}_{j}^{m}, k \neq i, h \in \mathcal{T}_{k}^{m}\right) .
\end{aligned}
$$

In addition, for successful scheduling in frequency domain, the following two constraints must also hold:

(C-4) For a band $m \in \mathcal{M}_{j}$ that is available at node $j$, this band cannot be used for both transmission and receiving. That is, if band $m$ is used at node $j$ for transmission (or receiving), then it cannot be used for receiving (or transmission).

(C-5) Similar to constraint (C-3) on transmission, node $j$ cannot use the same band $m \in \mathcal{M}_{j}$ to receive from two different nodes.

Note that (C-4) can be viewed as "self-interference" avoidance constraint where at the same node $j$, its transmission to another node $h$ on band $m$ interferes its reception from node $i$ on the same band. It turns out that the above two constraints are mathematically embedded in (C-1') and (C-2'). That is, once (C-1') and (C-2') are satisfied, then both constraints (C$4)$ and (C-5) are also satisfied. This result is formally stated in the following lemma.

Lemma 1: If transmission powers on every transmission link and interference link satisfy (C-1') and (C-2') in the network, then (C-4) and (C-5) are also satisfied.

Proof. We first prove that (C-1') and (C-2') lead to (C-4). To do this, we let $k=j$ in (C-2'). Then (C-2') degenerates into $p_{j h}^{m} \leq P-P x_{i j}^{m}$ since $d_{j j}=0$.

- Suppose node $j$ is receiving from node $i$ on band $m$, i.e., $x_{i j}^{m}=1$, then $p_{j h}^{m} \leq P-P x_{i j}^{m}=0$. Since $p_{j h}^{m} \geq$ $\left(\frac{d_{j h}}{R_{T}^{m a x}}\right)^{n} P x_{j h}^{m}$ from (C-1'), we have that $x_{j h}^{m}$ must be 0 . That is, if node $j$ is receiving from node $i$ on band $m$, then node $j$ cannot transmit to a node $h$ in the same band.

- Now suppose node $j$ is transmitting to node $h$ on band $m$, i.e., $x_{j h}^{m}=1$. We will show that this will lead to $x_{i j}^{m}=0$. This can be proved by contradiction. That is, if $x_{i j}^{m}=1$, then we have just proved in the above paragraph that $x_{j h}^{m}=0$. But this contradicts our initial assumption that $x_{j h}^{m}=1$. Therefore, $x_{i j}^{m}$ must be 0 . That is, if node $j$ is transmitting to node $h$ on band $m$, then node $j$ cannot use the same band for receiving from a node $i$.

Combining the above two results, we have shown that $(\mathrm{C}-4)$ holds.

We now prove that (C-1') and (C-2') also lead to (C$5)$. Again the proof is based on contradiction. Suppose that (C-5) does not hold. Then node $j$ can receive from two different nodes $i$ and $k$ on the same band $m$, i.e., $x_{i j}^{m}=1$ and $x_{k j}^{m}=1$. Note that link $k \rightarrow j$ can be viewed as an interfering link to link $i \rightarrow j$. This corresponds to letting $h=j$ in (C-2'). Then from (C-2'), since $x_{i j}^{m}=1$, we have $p_{k j}^{m} \leq\left(\frac{d_{k j}}{R_{I}^{m a x}}\right)^{n} P$. On the other hand, by (C-1'), we have $p_{k j}^{m} \geq\left(\frac{d_{k j}}{R_{T}^{\text {max }}}\right)^{n} P$. However, the above two inequalities cannot hold simultaneously (contradiction) since we have $R_{I}^{\max }>R_{T}^{\max }$. Thus, the initial assumption that (C-5) does not hold is incorrect and the proof is complete.

The significance of Lemma 1 is that since $(\mathrm{C}-4)$ and $(\mathrm{C}-5)$ are embedded in (C-1') and (C-2'), it is sufficient to consider constraints $\left(\mathrm{C}-1^{\prime}\right),\left(\mathrm{C}-2^{\prime}\right)$, and (C-3) for scheduling and power control.

\section{Flow Routing and Link Capacity Constraints}

In this study, we assume there is a set of $\mathcal{L}$ active user communication (unicast) sessions in the CRN. Denote $s(l)$ and $d(l)$ the source and destination nodes of session $l \in \mathcal{L}$ and $r(l)$ the rate requirement (in $\mathrm{b} / \mathrm{s}$ ) of session $l$. We consider the most general case of multi-path routing, i.e., we allow flow splitting between a source node and its destination node.

Mathematically, this can be easily modeled based on flow balance at each node. Denote $f_{i j}(l)$ the data rate on link $(i, j)$ that is attributed to session $l$, where $i \in \mathcal{N}, i \neq d(l), j \in \mathcal{T}_{i}=$ $\bigcup_{m \in \mathcal{M}_{i}} \mathcal{T}_{i}{ }^{m}, j \neq s(l) .{ }^{1}$ If node $i$ is the source node of session $l$, i.e., $i=s(l)$, then

$$
\sum_{j \in \mathcal{T}_{i}} f_{i j}(l)=r(l) .
$$

If node $i$ is an intermediate relay node for session $l$, i.e., $i \neq$ $s(l)$ and $i \neq d(l)$, then

$$
\sum_{j \in \mathcal{T}_{i}}^{j \neq s(l)} f_{i j}(l)=\sum_{k \in \mathcal{T}_{i}}^{k \neq d(l)} f_{k i}(l) .
$$

If node $i$ is the destination node of session $l$, i.e., $i=d(l)$, then

$$
\sum_{k \in \mathcal{T}_{i}} f_{k i}(l)=r(l) .
$$

It can be easily verified that once (5) and (6) are satisfied, (7) must also be satisfied. As a result, it is sufficient to have (5) and (6) in the formulation.

In addition to the above flow balance equations at each node $i \in \mathcal{N}$ for session $l \in \mathcal{L}$, the aggregated flow rates on each radio link cannot exceed this link's capacity. Under $p_{i j}^{m}$, we have

$$
\begin{aligned}
\sum_{l \in \mathcal{L}}^{s(l) \neq j, d(l) \neq i} f_{i j}(l) & \leq \sum_{m \in \mathcal{M}_{i j}} c_{i j}^{m} \\
& =\sum_{m \in \mathcal{M}_{i j}} W \log _{2}\left(1+\frac{g_{i j}}{\eta W} p_{i j}^{m}\right),
\end{aligned}
$$

where $\eta$ is the ambient Gaussian noise density.

\section{PRoblem Formulation}

Objective Function. In this paper, we consider how to minimize network resource usage to support a set of user sessions. Network resource usage can be defined in a number of ways, which typically includes bandwidth usage. However,

\footnotetext{
${ }^{1}$ For $f_{i j}(l)$, we set $i \neq d(l)$ to ensure that the destination node $d(l)$ will be a sink node and not transmit data to a relay node $i$. We also set $j \neq s(l)$ to ensure that a relay node $i$ will not transmit data back to the source node $s(l)$.
} 
as pointed out in [13], bandwidth usage can only characterize resource usage in spectrum, but cannot characterize the impact (i.e., interference) of radio transmission in space. For example, a node transmitting with the same channel bandwidth but with different power levels will produce different interference "footprint" areas. To account for both the spectrum usage and the impact of a $\mathrm{CR}$ in space dimension, the so-called bandwidth-footprint-product (BFP) metric was introduced in [13] and will also be adopted in this paper. Since each node in the network will use a number of bands for transmission and each band will have a certain footprint corresponding to its transmission power, our objective will be to minimize network-wide BFP, which is the sum of BFPs among all the nodes in the network, i.e.,

$$
\min \sum_{i \in \mathcal{N}} \sum_{m \in \mathcal{M}_{i}} \sum_{j \in \mathcal{T}_{i}{ }^{m}} W \cdot \pi\left(R_{I}\left(p_{i j}^{m}\right)\right)^{2} .
$$

In some sense, minimizing network-wide BFP can be viewed as minimizing "weighted" version of bandwidth usage, where the weight is the interference footprint area. Note that (9) is equivalent to minimize $\pi\left(R_{I}^{\max }\right)^{2} \sum_{i \in \mathcal{N}} \sum_{m \in \mathcal{M}_{i}}$ $\sum_{j \in \mathcal{T}_{i}^{m}} W\left(\frac{p_{i j}^{m}}{P}\right)^{2 / n}$. Since $\pi\left(R_{I}^{\text {max }}\right)^{2}$ is a constant factor, we can remove it from the objective function. We also point out that although BFP is used as objective function in this paper, those traditional utility-based objective functions (e.g. [14], [16], [21]) can also be used if needed.

Discretization of Transmission Powers. For power control, we allow the transmission power to be adjusted between 0 and $P$. In practice, the transmission power can only be tuned into a finite number of discrete levels between 0 and $P$. To model this discrete version of power control, we introduce an integer parameter $Q$ that represents the total number of power levels to which a transmitter can be adjusted, i.e., $0, \frac{1}{Q} P, \frac{2}{Q} P, \cdots, P$. Denote $q_{i j}^{m} \in\{0,1,2, \cdots, Q\}$ the integer power level for $p_{i j}^{m}$, i.e., $p_{i j}^{m}=\frac{q_{i j}^{m}}{Q} P$. Then (C-1'), (C-2'), and (8) can be re-written as follows:

$$
\begin{gathered}
q_{i j}^{m} \in\left[\left(\frac{d_{i j}}{R_{T}^{m a x}}\right)^{n} Q x_{i j}^{m}, Q x_{i j}^{m}\right], \\
q_{k h}^{m} \leq Q-\left[1-\left(\frac{d_{k j}}{R_{I}^{m a x}}\right)^{n}\right] Q x_{i j}^{m} \quad\left(k \in \mathcal{I}_{j}^{m}, k \neq i, h \in \mathcal{T}_{k}^{m}\right),(11) \\
\sum_{l \in \mathcal{L}}^{s(l) \neq j, d(l) \neq i} f_{i j}(l) \leq \sum_{m \in \mathcal{M}_{i j}} W \log _{2}\left(1+\frac{g_{i j} P}{\eta W Q} q_{i j}^{m}\right) .
\end{gathered}
$$

We can re-formulate (11) as follows. Note that by (C-3), there is at most one $h \in \mathcal{T}_{k}^{m}$ such that $x_{k h}^{m}=1$. As a result, based on (10), there is at most one $q_{k h}^{m}>0$ in $\sum_{h \in \mathcal{T}_{k}^{m}} q_{k h}^{m}$. Thus, (11) can be rewritten as

$$
\sum_{h \in \mathcal{T}_{k}^{m}} q_{k h}^{m} \leq Q-\left(1-\left(\frac{d_{k j}}{R_{I}^{\text {max }}}\right)^{n}\right) Q x_{i j}^{m} \quad\left(k \in \mathcal{I}_{j}^{m}, k \neq i\right) .
$$

Problem Formulation. Putting together the objective function and all the constraints for per-node based power control, scheduling, and flow routing, we have the following formulation.

$$
\operatorname{Min} \quad \sum_{i \in \mathcal{N}} \sum_{m \in \mathcal{M}_{i}} \sum_{j \in \mathcal{T}_{i}^{m}} W\left(\frac{q_{i j}^{m}}{Q}\right)^{2 / n}
$$

$$
\begin{gathered}
\sum_{j \in \mathcal{T}_{i}^{m}} x_{i j}^{m} \leq 1 \quad\left(i \in \mathcal{N}, m \in \mathcal{M}_{i}\right) \\
q_{i j}^{m}-\left(\frac{d_{i j}}{R_{T}^{m a x}}\right)^{n} Q x_{i j}^{m} \geq 0 \quad\left(i \in \mathcal{N}, m \in \mathcal{M}_{i}, j \in \mathcal{T}_{i}^{m}\right) \\
q_{i j}^{m}-Q x_{i j}^{m} \leq 0 \quad\left(i \in \mathcal{N}, m \in \mathcal{M}_{i}, j \in \mathcal{T}_{i}^{m}\right) \\
\sum_{h \in \mathcal{T}_{k}^{m}} q_{k h}^{m}+\left(1-\left(\frac{d_{k j}}{R_{I}^{m a x}}\right)^{n}\right) Q x_{i j}^{m} \leq Q \\
\left(i \in \mathcal{N}, m \in \mathcal{M}_{i}, j \in \mathcal{T}_{i}^{m}, k \in \mathcal{I}_{j}^{m}, k \neq i\right) \\
\sum_{l \in \mathcal{L}} f_{i j}(l) \neq j, d(l) \neq i \\
\sum_{m \in \mathcal{M}_{i j}} W \log _{2}\left(1+\frac{g_{i j} P}{\eta W Q} q_{i j}^{m}\right) \leq 0 \\
\sum_{j \in \mathcal{T}_{i}} f_{i j}(l)=r(l) \quad\left(i \in \mathcal{N}, j \in \mathcal{T}_{i}\right) \\
k \neq d(l) \\
\sum_{j \in \mathcal{T}_{i}} f_{i j}(l)-\sum_{k \in \mathcal{T}_{i}} f_{k i}(l)=0 \quad(l \in \mathcal{L}, i \in \mathcal{N}, i \neq s(l), d(l)) \\
x_{i j}^{m} \in\{0,1\}, q_{i j}^{m} \in\{0,1,2, \cdots, Q\} \quad\left(i \in \mathcal{N}, m \in \mathcal{M}_{i}, j \in \mathcal{T}_{i}^{m}\right) \\
f_{i j}(l) \geq 0 \quad\left(l \in \mathcal{L}, i \in \mathcal{N}, i \neq d(l), j \in \mathcal{T}_{i}, j \neq s(l)\right),
\end{gathered}
$$

where (12) and (13) come from (10), $W, g_{i j}, R_{T}^{\max }, R_{I}^{\max }, P$, $\eta, r(l)$, and $Q$ are all constants and $x_{i j}^{m}, q_{i j}^{m}$, and $f_{i j}(l)$ are all optimization variables.

This optimization problem is in the form of mixed-integer non-linear program (MINLP), which is NP-hard in general [8]. In the next section, we develop a unified (instead of decoupled) solution procedure based on the branch-and-bound framework [15] and the convex hull relaxation to solve this problem.

\section{A Solution Procedure}

\section{A. Overview}

Note that the difficulty of this optimization problem comes from the integer variables $x_{i j}^{m}$ 's and $q_{i j}^{m}$ 's. Once the values of all integer variables are determined, the values of remaining variables $f_{i j}(l)$ 's can be obtained by a linear program (LP). Thus, the key step to solve our problem is to narrow down the value sets and finally determine these values for integer variables. We find that the so-called branch-and-bound framework [15] is most effective in solving this problem. Under this approach, we aim to provide a $(1-\varepsilon)$ optimal solution, which is formally defined as follows.

Definition 1: For a minimization problem, denote $y^{*}$ the objective value of the optimal solution. A feasible solution with objective value $y$ is called a $(1-\varepsilon)$ optimal solution if we have $y^{*} \geq(1-\varepsilon) y$.

Initially, the value set for $x_{i j}^{m}$ 's and $q_{i j}^{m}$ 's are $x_{i j}^{m} \in\{0,1\}$ and $q_{i j}^{m} \in\{0,1,2, \cdots, Q\}$. By using some relaxation technique, branch-and-bound obtains a linear relaxation for the original MINLP problem and its solution provides a lower bound $(L B)$ to the objective function. As we shall show shortly, this critical step is made possible by the convex hull relaxation for non-linear discrete terms. With the relaxation solution as a starting point, branch-and-bound uses a local search algorithm to find a feasible solution to the original 
problem, which provides an upper bound $(U B)$ for the objective function. If the obtained lower and upper bounds are within $\varepsilon$ to each other, i.e., $L B \geq(1-\varepsilon) U B$, we are done.

If the relaxation errors are not small, then the upper bound $U B$ could be far away from the lower bound $L B$. To close this gap, we must have a tighter linear relaxation, i.e., with smaller relaxation errors. This could be achieved by further narrowing down the value sets of $x_{i j}^{m}$ 's and $q_{i j}^{m}$ 's. We call these variables as partition variables. Specifically, branch-andbound selects a partition variable with the largest relaxation error and divides its value set into two sets based on its value in the relaxation solution. For example, if a $q_{i j}^{m}$ with a value set $\{0,1,2,3\}$ is selected and its value in the relaxation solution is 1.3 , then its value set is divided into two sets $\{0,1\}$ (the set of values no more than 1.3) and $\{2,3\}$ (the set of values no less than 1.3). Then the original problem (denoted as problem 1 ) is divided into two new problems (denoted as problem 2 and problem 3). Again, branch-and-bound performs relaxation and local search on these two new problems. Now we have lower bounds $L B_{2}$ and $L B_{3}$ for problems 2 and 3 , respectively. We also have feasible solutions that provide upper bounds $U B_{2}$ and $U B_{3}$ for problems 2 and 3, respectively. Since the relaxations in problems 2 and 3 are both tighter than that in problem 1 , we have $\min \left\{L B_{2}, L B_{3}\right\} \geq L B_{1}$ and $\min \left\{U B_{2}, U B_{3}\right\} \leq U B_{1}$. For a minimization problem, the lower bound of the original problem is updated from $L B=L B_{1}$ to $L B=\min \left\{L B_{2}, L B_{3}\right\}$. Also, the upper bound of the original problem is updated from $U B=U B_{1}$ to $U B=\min \left\{U B_{2}, U B_{3}\right\}$, since the best feasible solution to the original problem is the solution with a smaller $U B_{i}$. As a result, we now have smaller gap between $U B$ and $L B$. If $L B \geq(1-\varepsilon) U B$, we are done. Otherwise, we choose a problem with the minimum lower bound and perform partition for this problem.

Note that during the process for branch-and-bound, if we find a problem $z$ with $L B_{z} \geq(1-\varepsilon) U B$, then we can conclude that this problem cannot contribute to find a $(1-\varepsilon)$ optimal solution. $^{2}$ We can thus remove this problem from list for further consideration. Eventually, we will find $L B \geq(1-$ $\varepsilon) U B$ and the branch-and-bound procedure terminates. It has been shown that under very general conditions, a branch-andbound solution procedure always converges [20]. Moreover, although the worst-case complexity of such a procedure is exponential, the actual running time could be fast when all partition variables are integers (e.g., the problem considered in this paper).

Figure 2 shows the pseudocode of branch-and-bound framework. There are several key components in this framework that are problem specific and need to be carefully designed. These components are described in the following sections.

\footnotetext{
${ }^{2}$ For such a problem $z$, there are two possible cases. Case 1: The global optimal solution is not in problem $z$. In this case, the removal of problem $z$ will not cause the loss of the optimal solution in future iterations. Case 2: The global optimal solution is in problem $z$. In this case, the optimal (feasible) solution must have an objective value greater than or equal to $L B_{z}$, which means that it is also greater than or equal to $(1-\varepsilon) U B$ (since $L B_{z} \geq$ $(1-\varepsilon) U B$ ). Thus, by Definition 1 , the current best feasible solution with objective value $U B$ is already a $(1-\varepsilon)$ optimal solution. Therefore, although the removal of problem $z$ may lead to the loss of an optimal solution, we can still guarantee $(1-\varepsilon)$ optimality, since we have already found a $(1-\varepsilon)$ optimal solution.
}

\section{Branch-and-bound Procedure}

Initialization:

1. Let the initial solution $\psi_{\varepsilon}=\emptyset$, the initial upper bound $U B=\infty$, and the initial problem list include only the original problem, denoted as problem 1 .

2. Determine initial value set for each partition variable.

3. Build a linear relaxation for problem 1 and obtain its solution $\hat{\psi}_{1}$.

4. The objective value of $\hat{\psi}_{1}$ is a lower bound $L B_{1}$ to problem 1.

Iteration:

5. Select problem $z$ that has the minimum $L B_{z}$ among all problems in the problem list.

6. Update lower bound by $L B=L B_{z}$.

7. Find a feasible solution $\psi_{z}$ from $\hat{\psi}_{z}$ via a local search algorithm and denote its objective value as $U B_{z}$

8. If $\left(U B_{z}<U B\right)\{$

9. Update $\psi_{\varepsilon}=\psi_{z}$ and $U B=U B_{z}$.

10. If $L B \geq(1-\varepsilon) U B$, stop with the $(1-\varepsilon)$ optimal solution $\psi_{\varepsilon}$.

Otherwise, remove all problems $z^{\prime}$ with $L B_{z^{\prime}} \geq$ $(1-\varepsilon) U B$ from the problem list. \}

11. Select a variable with the largest relaxation error and divide its value set into two sets based on its value in $\hat{\psi}_{z}$.

12. Build two new problems $z_{1}$ and $z_{2}$ based on these two sets.

13. Remove problem $z$ from the problem list.

14. Obtain $L B_{z 1}$ and $L B_{z 2}$ for problems $z_{1}$ and $z_{2}$ via their linear relaxations.

15. If $L B_{z 1} \leq(1-\varepsilon) U B$, add problem $z_{1}$ into the problem list.

16. If $L B_{z 2} \leq(1-\varepsilon) U B$, add problem $z_{2}$ into the problem list.

17. If the problem list is empty, we stop with the $(1-\varepsilon)$ optimal solution $\psi_{\varepsilon}$. Otherwise, go to the next iteration.

Fig. 2. Pseudocode for branch-and-bound.

\section{B. Linear Relaxation}

During each iteration of branch-and-bound, we need a relaxation technique to obtain a lower bound of the objective function (see line 4 in Fig. 2). For a non-linear discrete term, we propose to use a convex hull relaxation. That is, we introduce a new variable $u_{i j}^{m}$ for non-linear discrete term $\left(q_{i j}^{m}\right)^{2 / n}$. Suppose $q_{i j}^{m} \in\left\{q_{0}, q_{1}, \cdots, q_{K}\right\}$, where $\left(q_{i j}^{m}\right)_{L}=$ $q_{0}<q_{1}<\cdots<q_{K}=\left(q_{i j}^{m}\right)_{U}$. The convex hull (see Fig. 3) can be formulated as

$$
\begin{gathered}
u_{i j}^{m}-\frac{\left(q_{K}\right)^{2 / n}-\left(q_{0}\right)^{2 / n}}{q_{K}-q_{0}} q_{i j}^{m} \geq \frac{q_{K}\left(q_{0}\right)^{2 / n}-\left(q_{K}\right)^{2 / n} q_{0}}{q_{K}-q_{0}}, \\
u_{i j}^{m}-\frac{\left(q_{k}\right)^{2 / n}-\left(q_{k-1}\right)^{2 / n}}{q_{k}-q_{k-1}} q_{i j}^{m} \leq \frac{q_{k}\left(q_{k-1}\right)^{2 / n}-\left(q_{k}\right)^{2 / n} q_{k-1}}{q_{k}-q_{k-1}} \\
(1 \leq k \leq K) .
\end{gathered}
$$

Similarly, we can introduce a new variable $v_{i j}^{m}$ for non-linear discrete term $\log _{2}\left(1+\frac{g_{i j} P}{\eta W Q} q_{i j}^{m}\right)$ and construct convex hull constraints for $v_{i j}^{m}$.

Denote $\mathbf{x}$ and $\mathbf{q}$ as the vector for variables $x_{i j}^{m}$ and $q_{i j}^{m}$, respectively. We have the following linear relaxation for problem $z$.

Min $\quad \sum_{i \in \mathcal{N}} \sum_{m \in \mathcal{M}_{i}} \sum_{j \in \mathcal{T}_{i}^{m}} \frac{W}{Q^{-2 / n}} u_{i j}^{m}$ 


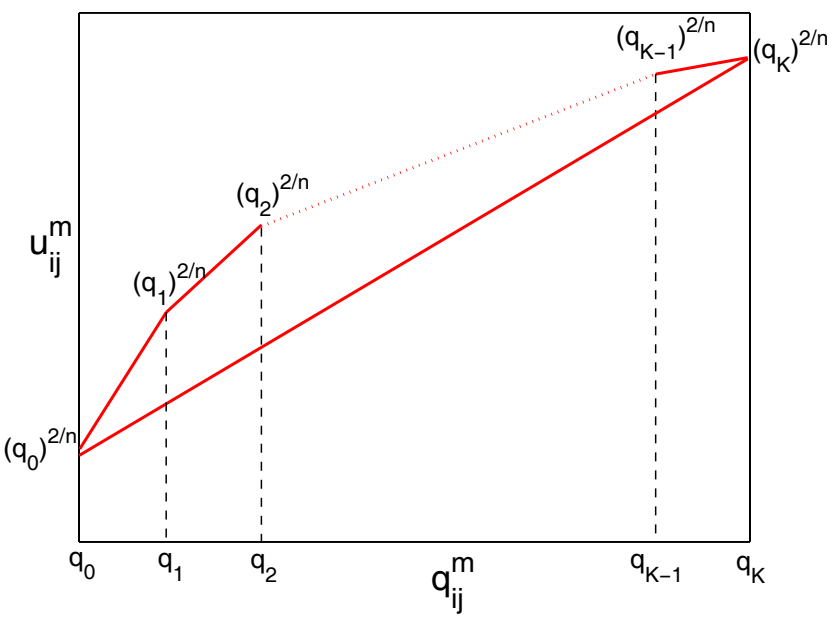

Fig. 3. Illustration of convex hull for a discrete term.

s.t. Convex hull constraints for $u_{i j}^{m} \quad\left(i \in \mathcal{N}, m \in \mathcal{M}_{i}, j \in \mathcal{T}_{i}^{m}\right)$

$$
\begin{array}{r}
\sum_{j \in \mathcal{T}_{i}^{m}} x_{i j}^{m} \leq 1 \quad\left(i \in \mathcal{N}, m \in \mathcal{M}_{i}\right) \\
q_{i j}^{m}-\left(\frac{d_{i j}}{R_{T}^{m a x}}\right)^{n} Q x_{i j}^{m} \geq 0 \quad\left(i \in \mathcal{N}, m \in \mathcal{M}_{i}, j \in \mathcal{T}_{i}^{m}\right) \\
q_{i j}^{m}-Q x_{i j}^{m} \leq 0 \quad\left(i \in \mathcal{N}, m \in \mathcal{M}_{i}, j \in \mathcal{T}_{i}^{m}\right) \\
\sum_{h \in \mathcal{T}_{k}^{m}} q_{k h}^{m}+\left(1-\left(\frac{d_{k j}}{R_{I}^{m a x}}\right)^{n}\right) Q x_{i j}^{m} \leq Q \\
\left(i \in \mathcal{N}, m \in \mathcal{M}_{i}, j \in \mathcal{T}_{i}^{m}, k \in \mathcal{I}_{j}^{m}, k \neq i\right)
\end{array}
$$

$$
\begin{array}{rc}
\sum_{l \in \mathcal{L}}^{s(l) \neq j, d(l) \neq i} f_{i j}(l)-\sum_{m \in \mathcal{M}_{i j}} W v_{i j}^{m} \leq 0 & \left(i \in \mathcal{N}, j \in \mathcal{T}_{i}\right) \\
\text { Convex hull constraints for } v_{i j}^{m} & \left(i \in \mathcal{N}, m \in \mathcal{M}_{i}, j \in \mathcal{T}_{i}^{m}\right) \\
\sum_{j \in \mathcal{T}_{i}} f_{i j}(l)=r(l) & (l \in \mathcal{L}, i=s(l)) \\
\sum_{j \in \mathcal{T}_{i}}^{k \neq d(l)} f_{i j}(l)-\sum_{k \in \mathcal{T}_{i}}^{k \neq c} f_{k i}(l)=0 \quad(l \in \mathcal{L}, i \in \mathcal{N}, i \neq s(l), d(l)) \\
u_{i j}^{m}, v_{i j}^{m} \geq 0 \quad\left(i \in \mathcal{N}, m \in \mathcal{M}_{i}, j \in \mathcal{T}_{i}^{m}\right) \\
f_{i j}(l) \geq 0 \quad\left(l \in \mathcal{L}, i \in \mathcal{N}, i \neq d(l), j \in \mathcal{T}_{i}, j \neq s(l)\right) \\
(\mathbf{x}, \mathbf{q}) \in \Omega_{z},
\end{array}
$$

where $\Omega_{z}$ is the set of all possible values of $(\mathbf{x}, \mathbf{q})$ in problem $z$. For example, $\Omega_{1}$ for the original problem 1 is $\{(\mathbf{x}, \mathbf{q}): 0 \leq$ $\left.x_{i j}^{m} \leq 1,0 \leq q_{i j}^{m} \leq Q\right\}$.

\section{Local Search Algorithm}

A linear relaxation for a problem $z$ can be solved in polynomial time. Denote the relaxation solution as $\hat{\psi}_{z}$, which provides a lower bound to problem $z$, although it may not be feasible. We now show how to obtain a feasible solution $\psi_{z}$ based on $\hat{\psi}_{z}$ (see line 7 in Fig. 2).

For the feasible solution $\psi_{z}$, we use the same routing solution as that in $\hat{\psi}_{z}$, i.e., let $\mathbf{f}=\hat{\mathbf{f}}$. We then need to determine the value for $\mathbf{x}$ and $\mathbf{q}$ in $\psi_{z}$ such that the routing solution $\mathbf{f}$ is feasible, i.e., (8) should hold for each link $i \rightarrow j$.

Initially, each $q_{i j}^{m}$ is set as the smallest value $\left(q_{i j}^{m}\right)_{L}$ in its value set and $x_{i j}^{m}$ is fixed to 0 or 1 if its value set only has

\section{Local Search Algorithm}

Initialization:

1. Set $q_{i j}^{m}=\left(q_{i j}^{m}\right)_{L}$ and fix $x_{i j}^{m}$ to 0 or 1 if its value set only has one element 0 or 1 , respectively.

2. Compute the capacity $\sum_{m \in \mathcal{M}_{i}} W \log _{2}\left(1+\frac{g_{i j} P}{\eta W Q} q_{i j}^{m}\right)$ and the requirement $\sum_{l \in \mathcal{L}}^{s(l) \neq j, d(l) \neq i} f_{i j}(l)$ for each link $i \rightarrow j$.

Iteration:

3. If there is no link with its requirement larger than its capacity, then a feasible solution is found.

4. Otherwise, among all the links having their requirements larger than their capacities, find link $i \rightarrow j$ that has the largest requirement.

5. Try to increase the capacity for link $i \rightarrow j$ as follows.

6. Increase $q_{i j}^{m}$ among active bands in the non-increasing order of $\hat{q}_{i j}^{m}$ and under the limitation that $q_{i j}^{m} \leq$ $\min \left\{\left\lceil\hat{q}_{i j}^{m}\right\rceil,\left(q_{i j}^{m}\right)_{U}\right\}$.

7. If the achieved capacity is insufficient, then try to use an available but currently unused band $m$ in the non-increasing order of $\hat{q}_{i j}^{m}$.

8. A band $m$ is available if for any transmitting node $k$ using this band, node $j$ is not in its interference range.

9. Increase $q_{i j}^{m}$ under the constraint $q_{i j}^{m} \leq \min \left\{\left\lceil\hat{q}_{i j}^{m}\right\rceil\right.$, $\left.\left(q_{i j}^{m}\right)_{U}\right\}$.

10. Also set $x_{i j}^{m}=1, x_{i h}^{m}=0$ for $h \in \mathcal{T}_{i}, h \neq j$, and let $\left(q_{k h}^{m}\right)_{U} \leq\left\lfloor\left(\frac{d_{k j}}{R_{I}^{\text {max }}}\right)^{n} Q\right\rfloor$ for $k \in \mathcal{I}_{j}^{m}, k \neq i, h \in \mathcal{T}_{k}^{m}$.

11. If the achieved capacity is still insufficient, increase $q_{i j}^{m}$ among active bands in non-increasing order of $\hat{q}_{i j}^{m}$ and under the constraint $q_{i j}^{m} \leq\left(q_{i j}^{m}\right)_{U}$.

12. Finally, if the achieved capacity is still insufficient, then link $i \rightarrow j$ cannot be satisfied.

Fig. 4. An algorithm to obtain $\mathbf{x}$ and $\mathbf{q}$

one element 0 or 1 , respectively. Based on these $q_{i j}^{m}$ 's, we can compute the capacity $\sum_{m \in \mathcal{M}_{i}} W \log _{2}\left(1+\frac{g_{i j} P}{\eta W Q} q_{i j}^{m}\right)$ for each link $i \rightarrow j$. The requirement on a link $i \rightarrow j$ is $\sum_{l \in \mathcal{L}}^{s(l) \neq j, d(l) \neq i} f_{i j}(l)$. For each link with a requirement larger than its capacity, we will try to satisfy (8) by increasing $q_{i j}^{m}$ under its value set limitation. After we do this for all links, then we can calculate the objective value of solution $\psi_{z}$. Otherwise, if the requirement of any link cannot be satisfied, then we fail to find a feasible solution and we set the objective value to $\infty$. The details of this local search algorithm is shown in Fig. 4.

\section{Selection of Partition Variables}

If the relaxation error for a problem $z$ is not small, the gap between its lower and upper bounds may be large. To narrow this gap, we generate two new sub-problems $z_{1}$ and $z_{2}$ from problem $z$. We hope that these two new problems have smaller relaxation errors. Subsequently, they may have tighter bounds for the objective function. To generate $z_{1}$ and $z_{2}$, we identify a partition variable based on its relaxation error (line 11 in Fig. 2). As we discussed in Section IV-A, these partition variables include all $\mathbf{x}$ and $\mathbf{q}$ variables.

Since $\mathbf{x}$ variables are more important than $\mathbf{q}$ variables, we first select one of $\mathbf{x}$ variables. In particular, for the relaxation solution $\hat{\psi}_{z}$, we choose an $x_{i j}^{m}$ with the largest relaxation error $\min \left\{\hat{x}_{i j}^{m}, 1-\hat{x}_{i j}^{m}\right\}$ among all $\mathbf{x}$ variables and let its value set 


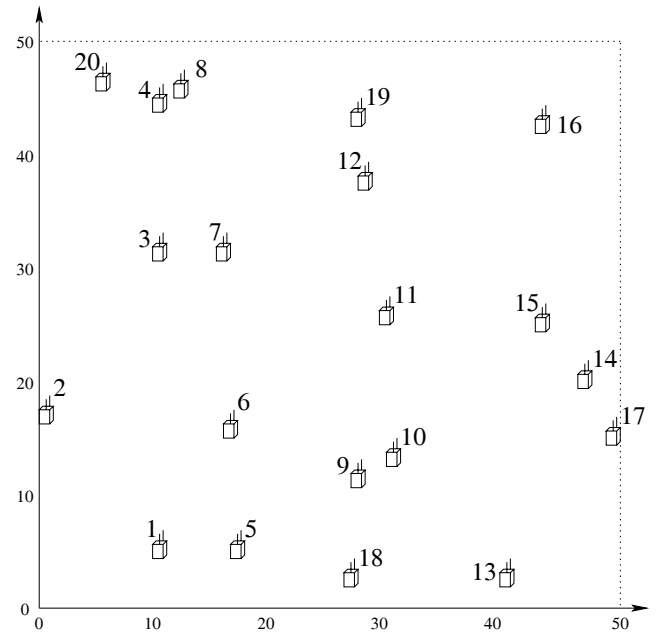

Fig. 5. A 20-node ad hoc network.

in problems $z_{1}$ and $z_{2}$ be $\{0\}$ and $\{1\}$, respectively. ${ }^{3}$ It should be note that the new value set of $x_{i j}^{m}$ imposes constraints on other variables. That is, if the new value set of $x_{i j}^{m}$ is $\{0\}$, then we have $q_{i j}^{m}=0$ based on (13). If the new value set of $x_{i j}^{m}$ is $\{1\}$, then we have $x_{i h}^{m}=0$ for $h \in \mathcal{T}_{i}, h \neq j$ based on (C-3), $q_{i j}^{m} \geq\left(\frac{d_{i j}}{R_{T}^{m a x}}\right)^{n} Q$ based on (12), and $q_{k h}^{m} \leq\left(\frac{d_{k j}}{R_{I}^{m a x}}\right)^{n} Q$ for $k \in \mathcal{I}_{j}^{m}, k \neq i, h \in \mathcal{T}_{k}$ based on (14). ${ }^{4}$

After we are done with all $\mathbf{x}$ variables, i.e., all $\mathbf{x}$ variables are fixed to either 0 or 1 , then we select one of $q$ variables. In particular, for the relaxation solution $\hat{\psi}_{z}$, the relaxation error of a discrete term $q_{i j}^{m}$ is $\min \left\{\hat{q}_{i j}^{m}-\left\lfloor\hat{q}_{i j}^{m}\right\rfloor,\left\lfloor\hat{q}_{i j}^{m}\right\rfloor+\right.$ $\left.1-\hat{q}_{i j}^{m}\right\}$; the relaxation error of a non-linear discrete term $u_{i j}^{m}=\left(q_{i j}^{m}\right)^{2 / n}$ is $\left|\hat{u}_{i j}^{m}-\left(\hat{q}_{i j}^{m}\right)^{2 / n}\right|$; and the relaxation error of a non-linear discrete term $v_{i j}^{m}=\log _{2}\left(1+\frac{q_{i j} P}{\eta W Q} q_{i j}^{m}\right)$ is $\left|\hat{v}_{i j}^{m}-\log _{2}\left(1+\frac{q_{i j} P}{\eta W Q} \hat{q}_{i j}^{m}\right)\right|$. Among these three types of relaxation errors, we identify the largest one and choose the corresponding $q_{i j}^{m}$ as the partition variable. Assuming the value set of $q_{i j}^{m}$ in problem $z$ is $\left\{q_{0}, q_{1}, \cdots, q_{K}\right\}$, its value set in problems $z_{1}$ and $z_{2}$ will be $\left\{q_{0}, q_{1}, \cdots,\left\lfloor\hat{q}_{i j}^{m}\right\rfloor\right\}$ and $\left\{\left\lfloor\hat{q}_{i j}^{m}\right\rfloor+1,\left\lfloor\hat{q}_{i j}^{m}\right\rfloor+2, \cdots, q_{K}\right\}$, respectively.

\section{NUMERICAl RESUlts}

\section{A. Simulation Setting}

In this section, we consider a randomly generated 20-node ad hoc network with each node located in a 50x50 area. For ease of exposition, we normalize all units for distance, bandwidth, rate, and power based on (1) and (8) with appropriate dimensions. An instance of network topology is given in Fig. 5 with each node's location listed in Table III. Within this network, we assume there are $|\mathcal{L}|=5$ user sessions, with source node and destination node randomly selected and the rate of each session is randomly generated within $[10,100]$. Table II specifies an instance of the source node, destination

\footnotetext{
${ }^{3}$ Since the value set for this $x_{i j}^{m}$ only has one element, this $x_{i j .}^{m}$ can be replaced by a constant in the new problem. As a result, some constraints may also be removed.

${ }^{4}$ If the constraints make the new value set of a variable $q_{k h}^{m}$ empty, then the corresponding new problem is clearly infeasible. As a result, we can remove it from the problem list.
}

TABLE II

SOURCE NODE, DESTINATION NODE, AND RATE REQUIREMENT OF THE 5 SESSIONS.

\begin{tabular}{|c|c|c|c|}
\hline Session & Source Node & Destination Node & Rate Requirement \\
\hline 1 & 7 & 16 & 28 \\
\hline 2 & 8 & 5 & 12 \\
\hline 3 & 15 & 13 & 56 \\
\hline 4 & 2 & 18 & 75 \\
\hline 5 & 9 & 11 & 29 \\
\hline
\end{tabular}

TABLE III

EACH NODE'S LOCATION AND AVAILABLE FREQUENCY BANDS FOR THE 20-NODE NETWORK.

\begin{tabular}{|c|c|c|}
\hline Node Index & Location & Available Bands \\
\hline 1 & $(10.5,4.3)$ & I, II, III, IV, V, VI, VII, VIII, IX, X \\
\hline 2 & $(1.7,17.3)$ & II, III, IV, V, VI, VII, X \\
\hline 3 & $(10.7,30.8)$ & I, III, IV, V, VI, VII, VIII, IX, X \\
\hline 4 & $(10.2,45.3)$ & I, III, IV, V, VI, VII, VIII, IX, X \\
\hline 5 & $(17.8,4)$ & I, II, V, VI, VII, VIII, IX \\
\hline 6 & $(17.2,15.2)$ & I, II, IV, VIII \\
\hline 7 & $(16.9,30.8)$ & I, II, III, IV, V, VI, VII, VIII, IX, X \\
\hline 8 & $(12.3,47.3)$ & I, III, IV, V, VII, VIII, IX \\
\hline 9 & $(28.2,11.5)$ & I, III, V, VII \\
\hline 10 & $(32.1,13.8)$ & I, II, III, IV, VI, VII, VIII, IX, X \\
\hline 11 & $(30.4,25.6)$ & I, II, III, V, VI, VIII, IX, X \\
\hline 12 & $(29.7,36)$ & I, II, III, IV, VI, VI \\
\hline 13 & $(41.7,3.1)$ & I, II, III, V, VI, VIII, IX, X \\
\hline 14 & $(47.5,20)$ & I, IV, V, VIII, IX, X \\
\hline 15 & $(43.3,25.3)$ & II, III, IV, V, VI, VII, VIII, IX, X \\
\hline 16 & $(44.1,42.7)$ & I, II, IV, VI, VII, VIII, IX, X \\
\hline 17 & $(49.6,15.8)$ & I, II, III, IV, V, VI, VII, VIII \\
\hline 18 & $(28.7,2.5)$ & I, II, III, VI, VII, VIII, IX, X \\
\hline 19 & $(28,43.5)$ & II, IV, V, VI, VIII \\
\hline 20 & $(5,46.9)$ & II, IV, V, VI, VII \\
\hline
\end{tabular}

node, and rate requirement for the five sessions in the network. We assume there are $|\mathcal{M}|=10$ frequency bands in the network and each band has a bandwidth of $W=50$. At each node, only a subset of these frequency bands is available. In the simulation, this is done by randomly selecting a subset of bands for each node. Table III shows the available bands for each node.

We assume $R_{T}^{\max }=20, R_{I}^{\max }=40$, and the path loss in$\operatorname{dex} n=4$. The threshold $\alpha$ is assumed to be $\alpha=\eta W=50 \eta$. Thus, we have $\beta=\left(\frac{R_{T}^{\max }}{R_{I}^{\max }}\right)^{n} \alpha W=\frac{50}{16} \eta$ and the maximum transmission power $P=\left(R_{T}^{\max }\right)^{n} \alpha W=8 \cdot 10^{6} \eta$. We set $\varepsilon=0.05$, which guarantees that the obtained solution is within $5 \%$ from the optimum.

\section{B. Results}

In this set of results, we apply the solution procedure to the 20-node network described above for different level of power control granularity $(Q)$. Note that $Q=1$ corresponds to the case that there is no power control, i.e., a node always uses its peak power $P$ for transmission. When $Q$ is sufficiently large, power control approaches continuous. Figure 6 shows the results. First, we note that power control has a significant impact on objective value. Comparing the case when there is 


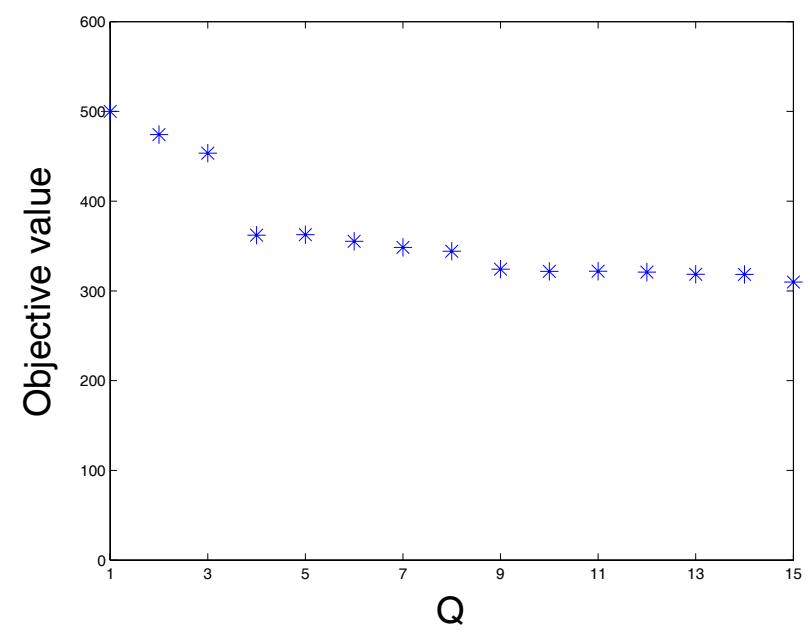

Fig. 6. Objective value as a function of $Q$.

no power control $(Q=1)$ and the case of $Q=15$, we find that there is nearly a $40 \%$ reduction in the objective value. Second, although the objective value is a non-increasing function of $Q$, when $Q$ becomes sufficiently large (e.g., 10 in this network setting), further increase in $Q$ will not have much reduction on objective value. This suggests that for practical purpose, the number of required power control levels does not need to be a large number.

The rest of results are for $Q=10$. For transmission power, we have

$q_{9,11}^{1}=3$

$q_{2,1}^{4}=4$,

$q_{12,16}^{2}=4$,

$q_{2,1}^{5}=4$,

$q_{17,13}^{7}=4$,

$q_{8,3}^{8}=5, q_{14,17}^{8}=1$,

$q_{3,2}^{10}=5$.

$q_{7,12}^{3}=3$,

$q_{5,18}^{6}=2$,

$q_{1,5}^{9}=1, q_{15,14}^{9}=1$,

The scheduling results are

$x_{9,11}^{1}=1, \quad x_{12,16}^{2}=1$,

$x_{2,1}^{4}=1, \quad x_{2,1}^{5}=1$,

$x_{17,13}^{7,1}=1, \quad x_{8,3}^{8}=1, x_{14,17}^{8}=1, \quad x_{1,5}^{9}=1, x_{15,14}^{9}=1$,

$x_{3,2}^{10}=1$.

The flow routing topology is shown in Fig. 7. The corresponding flow rates are

$f_{7,12}(1)=28, \quad f_{12,16}(1)=28$,

$f_{8,3}(2)=12, \quad f_{3,2}(2)=12, \quad f_{2,1}(2)=12, \quad f_{1,5}(2)=12$,

$f_{15,14}(3)=56, \quad f_{14,17}(3)=56, \quad f_{17,13}(3)=56$,

$f_{2,1}(4)=75, \quad f_{1,5}(4)=75, \quad f_{5,18}(4)=75$,

$f_{9,11}(5)=29$.

Note that a link may be used by multiple sessions. For example, link $2 \rightarrow 1$ is used by sessions 2 and 4 . As a result, the total data rate on link $2 \rightarrow 1$ is $f_{2,1}(2)+f_{2,1}(4)=$ $12+75=87$.

The following observations on the numerical results show the close coupling relationships between per-node power control and upper layers. In one observation (scheduling), we can see that, links $8 \rightarrow 3$ and $14 \rightarrow 17$ are active on the same band 8 . This is feasible because the interference range at node 14 is 22.49 under $q_{14,17}^{8}=1$, which is smaller than 38.35 (the distance between nodes 3 and 14). We note that if there is no power control, i.e., node 14 use the peak transmission power, then node 3 will be in the interference range of node 14 ,

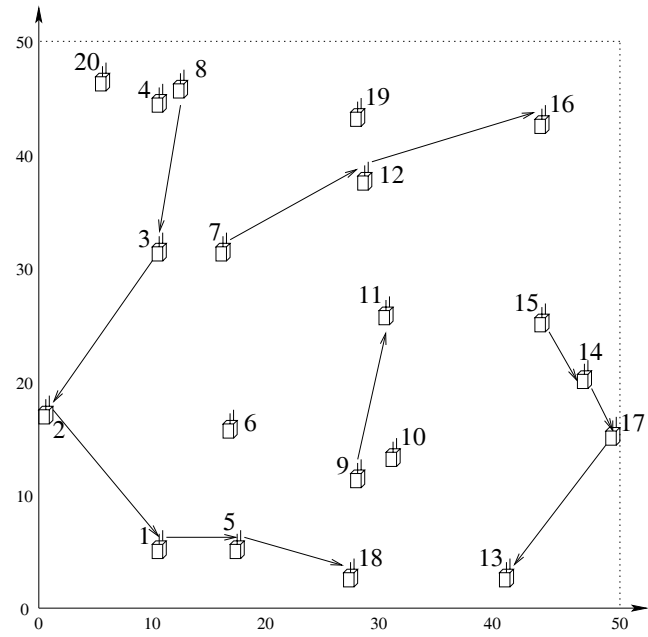

Fig. 7. Flow routing for the five sessions in the 20-node network.

which is $R_{I}^{\max }=40$, and will lead to a scheduling conflict. In another observation (routing), we see that, for session 2 (from node 8 to node 5 ), the routing path is $8 \rightarrow 3 \rightarrow 2 \rightarrow 1 \rightarrow 5$. Here, a shorter path $8 \rightarrow 3 \rightarrow 6 \rightarrow 5$ is not used. This is because that path $8 \rightarrow 3 \rightarrow 6 \rightarrow 5$ is interfered by transmissions on other paths. The optimal solution tends to choose paths that are not close to each other. Finally, for session 3 (form node 15 to node 13), the routing path is $15 \rightarrow 14 \rightarrow 17 \rightarrow 13$, while a shorter path $15 \rightarrow 17 \rightarrow 13$ is not used. This is because that node 15 can use a smaller transmission power to transmit to a closer neighboring node 14 with a smaller interference footprint. This will allow link $1 \rightarrow 5$ to be active on the same band 9 with link $15 \rightarrow 14$.

\section{CONCLUSION}

In this paper, we investigated the challenging problem of per-node based power control for a multi-hop CRN. This problem is difficult due to its large design space and the coupling relationship between power control and upper layers. We developed a mathematical framework to optimize these layers jointly and formulated a mixed integer non-linear program. Subsequently, we developed a solution procedure to derive results for all three layers jointly. Our solution guarantees $(1-\varepsilon)$ optimal performance. The results in this paper close some long standing issues associated with network optimization with per-node based power control.

\section{REFERENCES}

[1] M. Alicherry, R. Bhatia, and L. Li, "Joint channel assignment and routing for throughput optimization in multi-radio wireless mesh networks," in Proc. ACM Mobicom, pp. 58-72, Cologne, Germany, Aug. 2005.

[2] A. Behzad and I. Rubin, "Impact of power control on the performance of ad hoc wireless networks," in Proc. IEEE Infocom, pp. 102-113, Miami, FL, Mar. 2005.

[3] R. Bhatia and M. Kodialam, "On power efficient communication over multi-hop wireless networks: joint routing, scheduling and power control," in Proc. IEEE Infocom, pp. 1457-1466, Hong Kong, China, Mar. 2004.

[4] C. C. Chen and D. S. Lee, "A joint design of distributed QoS scheduling and power control for wireless networks," in Proc. IEEE Infocom, 12 pages, Barcelona, Catalunya, Spain, Apr. 2006.

[5] R. L. Cruz and A. V. Santhanam, "Optimal routing, link scheduling and power control in multi-hop wireless networks," in Proc. IEEE Infocom, pp. 702-711, San Francisco, CA, Mar. 2003. 
[6] R. Draves, J. Padhye, and B. Zill, "Routing in multi-radio, multihop wireless mesh networks," in Proc. ACM Mobicom, pp. 114-128, Philadelphia, PA, Sept. 2004.

[7] T. Elbatt and A. Ephremides, "Joint scheduling and power control for wireless ad-hoc networks," in Proc. IEEE Infocom, pp. 976-984, New York, NY, June 2002.

[8] M. R. Garey and D. S. Johnson, Computers and Intractability: A Guide to the Theory of NP-Completeness. New York: W.H. Freeman and Company, pp. 245-248, 1979.

[9] P. Gupta and P. R. Kumar, "The capacity of wireless networks," IEEE Trans. Inform. Theory, vol. 46, no. 2, pp. 388-404, Mar. 2000.

[10] Joint Tactical Radio System. [Online]. Available: http://jtrs.army.mil/.

[11] M. Kodialam and T. Nandagopal, "Characterizing the capacity region in multi-radio multi-channel wireless mesh networks," in Proc. ACM Mobicom, pp. 73-87, Cologne, Germany, Aug. 2005.

[12] P. Kyasanur and N. H. Vaidya, "Capacity of multi-channel wireless networks: impact of number of channels and interfaces," in Proc. ACM Mobicom, pp. 43-57, Cologne, Germany, Aug. 2005.

[13] X. Liu and W. Wang, "On the characteristics of spectrum-agile communication networks," in Proc. IEEE DySpan, pp. 214-223, Baltimore, MD, Nov. 2005.

[14] J. Liu, T. Y. Park, Y. T. Hou, Y. Shi, and H. D. Sherali, "Crosslayer optimization of MIMO-based mesh networks under orthogonal channels," in Proc. IEEE Wireless Commun. Networking Conf. (WCNC), pp. 49-54, Hong Kong, China, Mar. 2007.

[15] G. L. Nemhauser and L. A. Wolsey, Integer and Combinatorial Optimization. New York: John Wiley \& Sons, 1999.

[16] B. Radunovic and J.-Y. Le Boudec, "Rate performance objectives of multi-hop wireless networks," in Proc. IEEE Infocom, pp. 1916-1927, Hong Kong, China, Mar. 2004.

[17] A. Raniwala and T. Chiueh, "Architecture and algorithms for an IEEE 802.11-based multi-channel wireless mesh network," in Proc. IEEE Infocom, pp. 2223-2234, Miami, FL, Mar. 2005.

[18] A. Wyglinski, M. Nekovee, and Y. T. Hou, eds., Cognitive Radio Communications and Networks: Principles and Practice. Elsevier, 2009.

[19] SDR Forum. [Online]. Available: http://www.sdrforum.org.

[20] H. D. Sherali and W. P. Adams, A Reformulation-Linearization Technique for Solving Discrete and Continuous Nonconvex Problems, Chapter 8. Kluwer Academic Publishers, 1999.

[21] Y. Shi, Y. T. Hou, H. D. Sherali, and S. Kompella, "Cross-layer optimization for UWB-based ad hoc networks," in Proc. IEEE Military Commun. Conf. (Milcom), pp. 1-7, Washington, DC, Oct. 2006.

[22] Y. Shi and Y. T. Hou, "Optimal power control for multi-hop software defined radio networks," in Proc. IEEE Infocom, pp. 1694-1702, Anchorage, AK, May 2007.

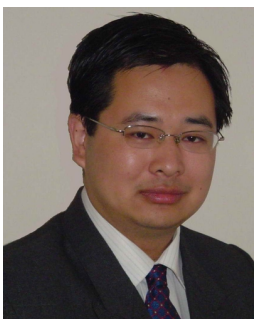

Yi Shi (S'02-M'08) received his B.S. degree from University of Science and Technology of China, Hefei, China, in 1998, a M.S. degree from Institute of Software, Chinese Academy of Science, Beijing, China, in 2001, a second M.S. degree from Virginia Polytechnic Institute and State University (Virginia Tech), Blacksburg, VA, in 2003, all in computer science, and a Ph.D. degree in computer engineering from Virginia Tech, in 2007. He is currently a Research Scientist in the Department of Electrical and Computer Engineering at Virginia Tech.

Dr. Shi's current research focuses on algorithms and optimization for cognitive radio wireless networks, MIMO and cooperative communication networks, sensor networks, and ad hoc networks. He was a recipient of IEEE INFOCOM 2008 Best Paper Award. He was a recipient of Chinese Government Award for Outstanding Ph.D. Students Abroad in 2006. While an undergraduate, he was a recipient of Meritorious Award in International Mathematical Contest in Modeling in 1997 and 1998, respectively. He was a TPC member of many major international conferences.

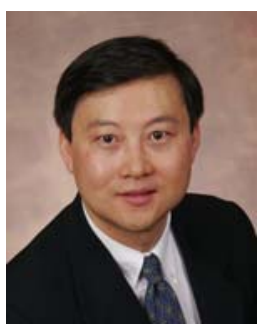

Y. Thomas Hou (S'91-M'98-SM'04) received his B.E. degree from the City College of New York in 1991, M.S. degree from Columbia University in 1993, and Ph.D. degree from Polytechnic Institute of New York University (formerly known as Polytechnic University) in 1998, all in Electrical Engineering. From 1997 to 2002, Dr. Hou was a Researcher at Fujitsu Laboratories of America, Sunnyvale, CA. Since 2002, he has been with Virginia Polytechnic Institute and State University (Virginia Tech), the Bradley Department of Electrical and Computer Engineering, Blacksburg, VA, where he is now an Associate Professor.

Prof. Hou's research interests are cross-layer design and optimization for MIMO-based ad hoc networks, cognitive radio wireless networks, cooperative communications, video communications over dynamic ad hoc networks, and algorithm design for sensor networks. He is a recipient of an Office of Naval Research (ONR) Young Investigator Award (2003) and a National Science Foundation (NSF) CAREER Award (2004) for his research on optimizations and algorithm design for wireless ad hoc and sensor networks. He has published extensively in leading IEEE and ACM journals and top-tier IEEE and ACM conferences and received five best paper awards from IEEE (including IEEE INFOCOM 2008 Best Paper Award and IEEE ICNP 2002 Best Paper Award). He holds five U.S patents.

Prof. Hou is currently serving as an Editor of IEEE TRANSACTIONS ON WiRELESS COMMUNICATIONS, IEEE WiRELESS COMMUNICATIONS MAGAZINE, ACM/SPRINGer Wireless NetWorks (WINET), and ElSEVIER AD Hoc NETwORKS. He was a past Associate Editor of IEEE Transactions on Vehicular Technology. He was Founding Chair of the First IEEE Workshop on Networking Technologies for Software Defined Radio Networks, September 25, 2006, Reston, VA. He was TPC Co-Chair of IEEE INFOCOM 2009 in Rio de Janeiro, Brazil.

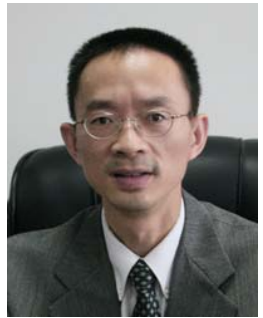

Huaibei (Barry) Zhou received his B.S and M.S degrees in Physics from Wuhan University, China in 1984 and 1987, respectively, and his Ph.D. degree in Physics from Univ. of Maryland, College Park, in 1994. His previous experience includes Postdoctoral Fellow at National Institute of Standard and Technology (NIST) in Maryland (1994-1996), Senior Engineer at GE (1996-1999) and Senior Technical Manager at NEXTEL (1999-2002). He is currently Dean of International School of Software Engineering, Wuhan University, China. His current research interests include cognitive radio and radio wave propagation. 